\section{Duodenal Variceal Bleeding Treated with a Transjugular Intrahepatic Portosystemic Shunt}

Most cases of upper gastrointestinal bleeding in patients with portal hypertension are caused by esophagogastric varices. Less often, bleeding originates in varices located elsewhere (1). If ectopic varices are found, the same hemostatic technique tend to be used. However, there is no evidence that such techniques are useful in these cases. A 25 -year-old, cirrhotic patient was admitted because of melena. On admission, his hematocrit level was $18 \%$. An emergency endoscopy showed large, nonbleeding esophageal varices and a voluminous duodenal varix with a clot marking the site of bleeding. Despite treatment with somatostatin, the patient rebled 24 hours after admission. A new endoscopy confirmed that the duodenal varix was reponsible for the recurrence, and sclerotherapy was performed with $14 \mathrm{ml}$ of $5 \%$ ethanolamine. Nevertheless, the transfusion of ten packs of red blood cells was required during the following 24 hours when ascites, encephalopathy, and jaundice developed. The patient was considered as a candidate for a transjugular intrahepatic portosystemic shunt (TIPS) because of his poor surgical risk. A self expandible prosthesis ("Wall Stent") succeeded in reducing the portosystemic gradient from 21 to $7 \mathrm{mmHg}$. Once the prosthesis had been placed, portography (Figure 1) showed that portal blood no longer filled the collateral vein feeding the duodenal varix. Six months later the portosystemic gradient remains unchanged.

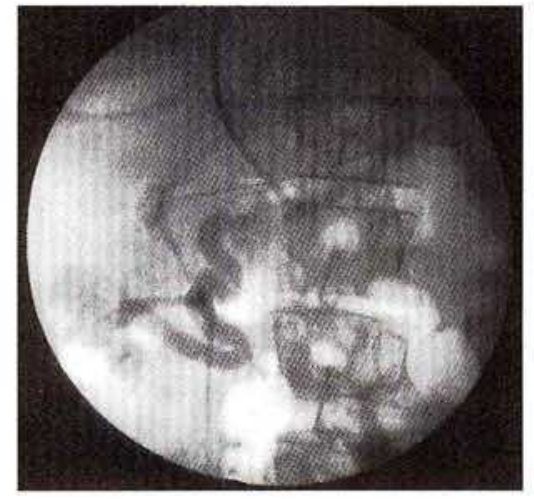

Figure 1: Retrograde portography after transjugular portosystemic shunt showing the large duodenal varix which caused the bleeding.

Duodenal varices are quite common, although they rarely bleed due to their location deep in the duodenal wall $(2,3)$. Consequently, if emergency endoscopy is not conducted, hemorrhage may be wrongfully attributed to coexisting gastroesophageal varices in a patient with portal hypertension without active bleeding. When bleeding is due to duodenal varices, two reasons may account for the controversial treatment: first, few cases have been reported and consequently different treatments cannot be compared; and second, in contrast to esophagogastric varices duodenal varices are retroperitoneal and drain into the inferior vena cava via the lumbar veins (1). This fact explains some hemodynamic differences between the two kinds of varices. Injection of vasopressin into the mesenteric artery has led to contradictory results $(1-3)$. Embolization of 
duodenal varices may be dangerous because of migration of the embolism to the lungs. Moreover, it is not a long-term treatment since follow-up has shown either recanalization of obliterated veins or the development of collaterals. Sclerotherapy has been considered useful especially in Child B and C patients (4), but sclerosis may be dangerous due to the higher pressure of the blood circulating in these varices. Portal shunt surgery is the most effective method to obtain complete hemostasia, although mortality is increased with advanced liver disease (1). Such patients may benefit from a TIPS. The stent is introduced via the transjugular route joining the portal vein with one of the hepatic veins, thus reducing portal pressure. This technique is associated with lower morbidity and mortality than those of surgical portosystemic shunts, presenting a clear advantage in patients with advanced liver disease. However, definitive results about its effectiveness are lacking.

P. Sort, I. Elizalde, I. Llach, F. Feu, J. C. Garcia-Pagan, J. M. Salmeron, A. Mas, J. Bosch, J. M. Bordas, J. Rodes Liver Unit, Hospital Clinic de Barcelona, Barcelona, Spain

\section{References}

1. Khougeer F, Morrow C, Jordn P: Duodenal varices as a cause of upper gastrointestinal bleeding. Surgery 1987; 102: 548552.

2. Sauerbruch T, Weinzierl W, Dietrich HP, et al.: Sclerotherapy of bleeding duodenal varix. Endoscopy 1982; 14: 187-189.

3. Barbish $A W$, Ehrinpreis $M N$ : Successful injection therapy of bleeding duodenal varx. Am J Gastroenterol 1993; 88: 90-92. 4. Jimenez Sáenz M, Pallarés Manrique H, Romero Castro R, et al.: Hemorragia digestiva por varices duodenales. Rev Esp Enferm Dig 1994; 85: 209-211.

Corresponding Author

J. M. Bordas, M.D.

Gastrointestinal Endoscopy Section

Hospital Clinic

Villarroel 170 